PROCEEDINGS OF THE

AMERICAN MATHEMATICAL SOCIETY

Volume 128, Number 7, Pages 1911-1915

S 0002-9939(00)05236-9

Article electronically published on February 25, 2000

\title{
ENDOMORPHISMS OF THE PLANE SENDING LINEAR COORDINATES TO COORDINATES
}

\author{
CHARLES CHING-AN CHENG AND ARNO VAN DEN ESSEN
}

(Communicated by Wolmer V. Vasconcelos)

\begin{abstract}
Let $k$ be a field of characteristic zero. We show that an endomorphism of $k\left[X_{1}, X_{2}\right]$ which sends each linear coordinate to a coordinate is an automorphism of $k\left[X_{1}, X_{2}\right]$.
\end{abstract}

In 3 the authors raised the following question (referred to as Problem 1): let $k$ be a field of characteristic zero and $A:=k\left[X_{1}, \cdots, X_{n}\right]$ the polynomial ring over $k$. Is every $k$-endomorphism of $A$ which sends each coordinate of $A$ to a coordinate of $A$ an automorphism of $A$ ? (A polynomial $f$ of $A$ is called a coordinate of $A$ if there exist $F_{2}, \cdots, F_{n}$ in $A$ such that $A=k\left[f, F_{2}, \cdots, F_{n}\right]$.) Problem 1 was answered affirmatively for $n=2$ in [3]. The case $n \geq 3$ remains open for arbitrary $k$. However, as was observed by Derksen, a negative answer to Problem 1 for algebraically closed fields would give a counterexample to the Jacobian Conjecture. More explicitly, he shows that (3. Lemma 2.4]) if a $k$-endomorphism $\varphi$ of $A$ sends linear coordinates to coordinates, then $\operatorname{det} J \varphi(x) \neq 0$ for all $x \in k^{n}$, so $\operatorname{det} J \varphi \in k^{*}$ if $k$ is algebraically closed! (A polynomial in $A$ is called a linear coordinate if it is of the form $c_{1} X_{1}+\cdots+c_{n} X_{n}$ for some $c_{i}$ in $k$, not all zero.) Based on Derksen's Lemma, Jelonek 7] gives a positive answer in any dimension to Problem 1 for algebraically closed fields! (He shows that the coordinate preservation property implies that $\varphi$ is proper which together with $\operatorname{det} J \varphi \in k^{*}$ shows that $\varphi$ is an automorphism of $A$.)

In [10], Mikhalev, Yu and Zolotykh, motivated by Derksen's Lemma, consider the following stronger version of Problem 1, referred to as Problem 2: is every endomorphism of $A$ which sends every linear coordinate to a coordinate an automorphism? Of course (as observed above) in case that $k$ is an algebraically closed field the hypothesis implies that det $J \phi \in k^{*}$. So if the Jacobian Conjecture is true, the answer to Problem 2 is yes. Consequently, a negative solution would give a counter-example to the Jacobian Conjecture. For $n=2$ the algebraically closed case was solved affirmatively in $[3$. The case $n \geq 3$ remained open (if $k=\bar{k}$ ).

On the other hand, in [10] the authors show that the answer to Problem 2 is negative in case $n \geq 3$ and $k$ is any non-algebraically closed field. However, the case $n=2$ remained open for $k$ a non-algebraically closed field.

Received by the editors December 12, 1997 and, in revised form, September 1, 1998.

1991 Mathematics Subject Classification. Primary 13B25, 13F20, 14E09, 16 W20.

Key words and phrases. Jacobian conjecture, automorphism, endomorphism, derivation, algebraically closed field, coordinate. 
Now the remarkable point is that (in spite of the negative result for $n \geq 3$ ) the answer is yes for all fields $k$ ( $\operatorname{char} k=0$ in case $n=2$ ). This is the main result of this paper (Theorem 1.1). The importance of this seemingly marginal result (compared with [3], [7] and [10]) is that it shows again the enormous difference between two-dimensional space and $n$-dimensional space, where $n \geq 3$.

Another example is the Markus-Yamabe Conjecture (Jacobian Conjecture for differential equations) which turned out to be true for all $C^{1}$-vector fields on $\mathbb{R}^{2}$ (see [4], [5], 6]) and false for all $n \geq 3$ even for polynomial vectorfields on $\mathbb{R}^{n}$. Also the existence of exotic $\mathbb{C}^{n}$ 's for $n \geq 3$ (i.e., smooth affine algebraic varieties of dimension $n$ homeomorphic to $\mathbb{C}^{n}$ but not algebraic isomorphic to $\mathbb{C}^{n}$ ), while they do not exist for $n=2$ (see [2] and [8]), shows the difference between $n=2$ and $n \geq 3$.

Summarizing: In light of the above considerations, the positive solution of Problem 2 in case $n=2$ should inspire the reader to look for a (possible negative) solution of Problem 2 for $n \geq 3$ and $k$ algebraically closed (which in turn has important implications for the Jacobian Conjecture!).

The proof of this result is based on a modified version of a result due to Rabier [11], where a useful characterization of a coordinate of $k[X, Y]$ is given. An easy proof of this modified version is given in Section 3.

\section{The MAin RESUlT}

Throughout this section, $k$ denotes a field of characteristic zero and $R:=k[X, Y]$ the polynomial ring in two variables over $k$. The main result of this paper is the following.

Theorem 1.1. Let $\varphi$ be a $k$-endomorphism of $R$ which sends each linear coordinate to a coordinate. Then $\varphi$ is an automorphism of $R$.

The proof of this theorem is based on the following proposition, a modified version of Theorem 2.1 in Rabier[11, whose proof will be given in Section 3 (see Proposition 3.1).

Proposition 1.2. Let $P$ be a coordinate of $R$ with $n:=\operatorname{deg}_{Y} P>0$ and let $D:=$ $P_{Y} \partial_{X}-P_{X} \partial_{Y}$. Then $D^{n}(X) \in k$.

Proof of Theorem 1.1. i) Let $P:=\varphi(X)$. So $P$ is a coordinate of $R$. Hence there exists an automorphism $\psi$ of $R$ with $\psi(X)=P$. Then $\psi^{-1} \varphi$ is an endomorphism of $R$ sending $X$ to $X$. Furthermore, if $f$ is a linear coordinate of $R$, then $\varphi(f)$ is a coordinate, whence $\psi^{-1} \varphi(f)$ is a coordinate too. Consequently, replacing $\varphi$ by $\psi^{-1} \varphi$ we may assume that $\varphi(X)=X$.

ii) So let $\varphi(X)=X$ and $g=\varphi(Y)$. The hypothesis implies that $g-c X=$ $\varphi(Y-c X)$ is a coordinate of $R$ for all $c \in k$. It follows easily that $n:=\operatorname{deg}_{Y} g>0$. Write $g=g_{n} Y^{n}+g_{n-1} Y^{n-1}+\cdots+g_{1} Y+g_{0}$, with $g_{i} \in k[X]$ for all $i$. Since $g$ is a coordinate, it follows from Corollary 14 of [9] that its Newton polygon is a triangle and, consequently, $g_{n} \in k^{*}$. Replacing $Y$ by $Y-\frac{1}{n} \frac{g_{n-1}}{g_{n}}$, we may assume that $g_{n-1}=0$. Replacing $g$ by $g_{n}^{-1} g$, we may also assume that $g_{n}=1$. In the remainder of this proof we will show that $n=1$ (which concludes the proof, since if $n=1$, then $\varphi=\left(X, g_{1} Y+g_{0}\right)$ with $g_{1} \in k^{*}$, so $\varphi$ is an automorphism).

iii) So from now on we assume that $n \geq 2, g_{n}=1$ and $g_{n-1}=0$, and we will derive a contradiction. Since $g-c X$ is a coordinate of $R$ for all $c \in k$, it follows 
from Proposition 1.2 that

$$
\left(D+c \partial_{Y}\right)^{n}(X) \in k
$$

for all $c \in k$ where $D:=g_{Y} \partial_{X}-g_{X} \partial_{Y}$. Let $T$ be a new variable and consider the polynomial

$$
H(T):=\left(D+T \partial_{Y}\right)^{n}(X) \in k[X, Y][T] .
$$

It follows from (1.1) and Lemma 1.3 below that $H(T) \in k[T]$. In particular, $A:=$ the $T^{n-2}$-coefficient of $H(T)$ belongs to $k$. From Proposition 2.1 of Section 2, we deduce that $g_{X} \in k$. Consequently, $g=\lambda X+a(Y)$, for some $\lambda \in k$ and $a(Y) \in k[Y]$. However, $g-c X$ is a coordinate of $R$ for all $c \in k$. So, in particular, $a(Y)=g-\lambda X$ is a coordinate of $R$, a contradiction since $\operatorname{deg} a(Y)=n \geq 2$.

Lemma 1.3. Let $k$ be an infinite field and $f_{0}, \cdots, f_{n} \in k[X, Y]$. If $f(T):=$ $\sum f_{p} T^{p}$ (where $T$ is a new variable) is such that $f(c) \in k$ for all $c \in k$, then $f_{p} \in k$ for all $k$, i.e., $f(T) \in k[T]$.

Proof. Let $i, j \in \mathbf{N}$ with $i+j>0$, and let $f_{p, i, j}$ be the coefficient of the monomial $X^{i} Y^{j}$ appearing in $f_{p}$. Then the hypothesis implies that $\sum f_{p, i, j} c^{p}=0$ for all $c \in k$. Since $k$ is infinite, this implies that $f_{p, i, j}=0$ for all $p$ and all $i, j \geq 0$ with $i+j>0$, i.e., $f_{p} \in k$ for all $p$.

\section{A teChNiCAL PROposition}

Throughout this section we have the following notation: $g:=Y^{n}+g_{n-2} Y^{n-2}+$ $\cdots+g_{1} Y+g_{0} \in k[X, Y]$ with $n \geq 2$ and $g_{i} \in k[X]$ for all $i$. Put $D:=g_{Y} \partial_{X}-g_{X} \partial_{Y}$, $D(T):=D+T \partial_{Y}$, where $T$ is a new variable and $\partial:=\partial_{Y}$. Furthermore, define $D_{i}:=\partial^{i}\left(g_{Y}\right) \partial_{X}-\partial^{i}\left(g_{X}\right) \partial_{Y}$, for all $i \geq 0$. By Leibniz' rule (and induction on $k$ ) one obtains

$$
\partial^{k} D=\sum_{i=0}^{k}\left(\begin{array}{l}
k \\
i
\end{array}\right) D_{i} \partial^{k-i}
$$

for all $k \geq 0$. Let $A:=$ the $T^{n-2}$-coefficient of $D(T)^{n}(X)$. Since $D(T)^{n}(X)=$ $X(T)^{n-1}\left(G_{Y}\right)$, we obtain

$$
A=\sum_{k=0}^{n-2} \partial^{k} D \partial^{n-k-2}\left(g_{Y}\right)=\sum_{k=0}^{n-2} \partial^{k} D \partial^{n-k-1}(g) .
$$

So by (2.1) and (2.2) we get

$$
A=\sum_{k=0}^{n-2} \sum_{i=0}^{k}\left(\begin{array}{c}
k \\
i
\end{array}\right) D_{i} \partial^{n-i-1}(g) .
$$

Proposition 2.1. If $A \in k$, then $g_{X} \in k$.

Proof. i) We first show that $g_{j}^{\prime}=0$ for all $j \geq 1$. Suppose the contrary and let $j \geq 1$ be maximal such that $g_{j}^{\prime} \neq 0$. We will show that the $Y^{j}$-coefficient of $A$ is nonzero, contradicting that $A \in k$. Since $g_{X}=g_{j}^{\prime} Y^{j}+$ lower order $Y$-terms, one easily verifies that $\operatorname{deg}_{Y} D_{i} \partial^{n-i-1}(g) \leq j$, for all $0 \leq i \leq n-2$. By (2.3), 
$\operatorname{deg}_{Y} A \leq j$. So to show that the $Y^{j}$-coefficient of $A$ is nonzero it suffices to show that $\partial^{j} A \neq 0$. From (2.2) and (2.1) we deduce

$$
\begin{aligned}
\partial^{j} A & =\sum_{k=0}^{n-2} \partial^{j+k} D \partial^{n-k-1}(g) \\
& =\sum_{k=0}^{n-2}\left(\sum_{i=0}^{j+k}\left(\begin{array}{c}
j+k \\
i
\end{array}\right) D_{i} \partial^{j+k-i} \partial^{n-k-1}(g)\right) \\
& =\sum_{k=0}^{n-2} \sum_{i=0}^{j+k}\left(\begin{array}{c}
j+k \\
i
\end{array}\right) D_{i} \partial^{j+n-i-1}(g) .
\end{aligned}
$$

Furthermore, it is left to the reader to verify (using again that $g_{X}=g_{j}^{\prime} Y^{j}+$ lower order Y-terms) that

$$
D_{i}\left(\partial^{j+n-i-1}(g)\right)=0
$$

for $i \neq j$, and

$$
D_{j}\left(\partial^{n-1}(g)\right)=-j ! n ! g_{j}^{\prime}
$$

Consequently, $\partial^{j} A=\sum_{k=0}^{n-2}\left(\begin{array}{c}j+k \\ j\end{array}\right)(-j !) n ! g_{j}^{\prime} \neq 0$, as desired.

ii) By i) we get $g=a(Y)+g_{0}(X)$, where $a(Y)=Y^{n}+$ lower order $Y$-terms. Consequently, $D=a^{\prime}(Y) \partial_{X}-g_{0}^{\prime}(X) \partial_{Y}$ and by $(2.2)$

$$
\begin{aligned}
A & =\sum_{k=0}^{n-2} \partial^{k} D a^{(n-k-1)}(Y) \\
& =\sum_{k=0}^{n-2} \partial^{k}\left(-g_{0}^{\prime}(X) a^{(n-k)}(Y)\right) \\
& =-g_{0}^{\prime}(X)(n-1) a^{(n)}(Y) \\
& =-(n-1)(n !) g_{0}^{\prime}(X) .
\end{aligned}
$$

Since $n \geq 2$ and $A \in k$, this implies that $g_{0}^{\prime} \in k$, whence $g_{X} \in k$.

\section{A modification of a Result of Rabier}

In [11, Rabier proved the following result: let $P \in R:=k\left[X_{1}, X_{2}\right]$ with $\operatorname{deg} P=$ $n \geq 1$ and put $D_{P}:=P_{X_{2}} \partial_{X_{1}}-P_{X_{1}} \partial_{X_{2}}$. If $P$ is a coordinate of $R$, then $\left(D_{P}\right)^{n}\left(X_{1}\right)$ and $\left(D_{P}\right)^{n}\left(X_{2}\right)$ belong to $k$ and not both are zero. Furthermore, it is shown in [11] that the converse is true as well. In the proof of Theorem 1.1 we need the following modified version of the result mentioned above.

Proposition 3.1. Let $P$ be a coordinate of $R$ with $\operatorname{deg}_{X_{2}} P=n \geq 1$. Then $\left(D_{P}\right)^{n}\left(X_{1}\right) \in k *$.

Proof. Put $F_{1}:=P$ and let $\left(F_{1}, F_{2}\right)$ be an automorphism of $R$ with $\operatorname{det} J\left(F_{1}, F_{2}\right)=$ 1. Then $D_{P}=-\frac{\partial}{\partial F_{2}}$ and if $\left(G_{1}, G_{2}\right)$ denotes the inverse of $\left(F_{1}, F_{2}\right)$, then $X_{1}=$ $G_{1}\left(F_{1}, F_{2}\right)$. So

$$
\left(D_{P}\right)^{n}\left(X_{1}\right)=(-1)^{n}\left(\frac{\partial}{\partial F_{2}}\right)^{n} G_{1}\left(F_{1}, F_{2}\right)
$$


By (1.6) and (1.8) of [1] we have

$$
n=\operatorname{deg}_{X_{2}} F_{1}=\operatorname{deg}_{Y_{2}} G_{1}\left(Y_{1}, Y_{2}\right)=\operatorname{deg}_{F_{2}} G_{1}\left(F_{1}, F_{2}\right)
$$

or

$$
n=\operatorname{deg}_{F_{2}} G_{1}\left(F_{1}, F_{2}\right) .
$$

From (3.1) we deduce that $\left(D_{P}\right)^{n}\left(X_{1}\right)=(-1)^{n} n ! c$, where $c$ is the coefficient of $F_{2}^{n}$ in $G_{1}\left(F_{1}, F_{2}\right)$. By Corollary 1.4 [1], $c \in k^{*}$. So the desired result follows from $(3.2)$.

\section{ACKNOWLEDGEMENT}

The second author would like to thank the Department of Mathematical Sciences of Oakland University for the warm hospitality during his visit when this work was initiated.

\section{REFERENCES}

[1] K. Adjamagbo and A. van den Essen, A resultant criterion and formula for the inversion of a polynomial map in two variables, J. of Pure and Applied Algebra 64 (1990), 1-6. MR 91g:14011

[2] A. Choudary and A. Dimca, Complex Hypersurfaces diffeomorphic to affine spaces, preprint 1994. MR 95f:14084

[3] A. van den Essen and V. Shpilrain, Some combinatorial questions about polynomial mappings, J. of Pure and Applied Algebra 119 (1997), 47-52. MR 98g:13007

[4] R. Feßler, A proof of the two dimensional Markus-Yamabe stability conjecture, Annales Polonici Mathematici 62 (1995), 45-75.

[5] A. A. Glutsuk, The complete solution of the Jacobian problem for vector fields on the plane, Communications of the Moscow Mathematical Society, Russian Math. Surveys 49 (1994), 185-186, Translated in Uspekhi Mat. Nauk, 3 (1994).

[6] C. Gutierrez, A solution to the bidimensional global asymptotic stability conjecture, Ann. Inst. H. Poincaré Anal. Non Linéaire 12 (1995), 627-671. MR 96k:34099b

[7] Z. Jelonek, A solution of the problem of van den Essen and Shpilrain, IMUJ, preprint 1996 /19 Krakow.

[8] S. Kaliman, Exotic structures on $\mathbb{C}^{n}$ and $\mathbb{C}^{*}$-actions on $\mathbb{C}^{3}$, preprint 1994. MR 97e:14058

[9] J. H. McKay and S. S.-S. Wang, An inversion formula for two polynomials in two variables, J. of Pure and Applied Algebra 40 (1986), 245-257. MR 87j:12003

[10] A. Mikhalev, J. Yu and A. Zolotykh, Images of coordinate polynomials, Algebra Colloquium 4 (1997), 159-162, preprint 1997.

[11] P. Rabier, On components of polynomial automorphisms in two variables, Comm. Algebra 24 (1996), 929-937. MR 96m:14020

Department of Mathematics, Oakland University, Rochester, Michigan 48309

E-mail address: cheng@oakland.edu

Department of Mathematics, University of Nijmegen, Nijmegen, The Netherlands

E-mail address: essen@sci.kun.nl 